\title{
Smoking cessation knowledge, attitude and practices among tuberculosis physicians: A qualitative study
}

\author{
Arusyak Harutyunyan', Armine Abrahamyan', Zaruhi Grigoryan', Varduhi Hayrumyan', Nune Truzyan', Varduhi Petrosyan'
}

\begin{abstract}
INTRODUCTION Smoking cessation interventions within tuberculosis (TB) care are feasible, effective and efficient for increasing smoking cessation rates. We aimed to assess TB physicians' smoking cessation knowledge, attitude, and practices (KAP).

METHODS We conducted a qualitative study with 21 TB physicians and utilized directed deductive content analysis with predefined knowledge, attitude, and practice categories. Physicians' practice was analyzed using the ABC approach (Ask, Brief advice, and Cessation support).

RESULTS Physicians acknowledged the importance of quitting for improved treatment outcomes and decreased risk of TB relapse. Physicians revealed presumed drug interactions, possible side effects of pharmacotherapy, and reluctance to take additional medications as challenges of smoking cessation interventions. Physicians asked about smoking behavior and provided a brief quitting advice to TB patients; however, implementation of cessation support was limited due to poor knowledge of evidence-based cessation methods and the absence of formal tobacco dependence treatment algorithms within TB care.

CONCLUSIONS TB physicians' KAP on smoking cessation was limited. Interventions targeting physicians' knowledge and skills, and formalization of tobacco dependence treatment within TB care, are core for improving their smoking cessation practices in Armenia.
\end{abstract}

\section{AFFILIATION}

1 Turpanjian School of Public Health, American University of Armenia, Yerevan, Armenia

CORRESPONDENCE TO Arusyak Harutyunyan. Turpanjian School of Public Health, American University of Armenia, 40 Marshal Baghramyan Ave, 0019, Yerevan, Armenia. E-mail: aharutyunyan@ aua.am

\section{KEYWORDS}

$A B C$ approach, smoking cessation, tuberculosis, KAP, tobacco control, healthcare providers

Received: 30 July 2020 Revised: 11 November 2020 Accepted: 14 November 2020

\section{INTRODUCTION}

The association between active and passive exposure to tobacco smoke and the progression of tuberculosis (TB) and poor treatment outcomes is well established for several decades ${ }^{1-4}$. Globally, TB is one of the leading fatal diseases, whereas active smoking increases the risk of developing TB by 2-2.5 times and is significantly associated with TB mortality ${ }^{5}$. Armenia is a former Soviet Union country that faces the high dual burden of TB and tobacco. The smoking rate among the Armenian male population is one of the highest in the European region. Moreover, the smoking prevalence among adult male and female TB patients is even higher compared to the general population $(67.5 \%$ and $5.8 \%$ vs $53.4 \%$ and $2.3 \%$; respectively $)^{6}$. Around $20 \%$ of TB deaths in Armenia are attributed to smoking ${ }^{7}$.

Armenia was classified as a high-priority country for treating TB until $2016^{8}$. In 2018, the estimated TB incidence rate in Armenia was 31 per 100000 population, whereas the mortality rate was 1.3 per 100000 population ${ }^{9}$. Though the TB incidence rates have been declining during recent years, the increasing burden of drug-resistant TB remains alarming; in 2018, 20\% of incident TB cases in Armenia were drug-resistant ${ }^{9}$. Furthermore, in Armenia men take a higher dual burden of TB and tobacco, as in the case of a higher smoking rate, the men to women ratio of TB incidence is near $3: 1$, compared with the global ratio of $2: 1$ in the World 
Health Organization (WHO) European region, ${ }^{9,10}$.

In 2004, Armenia was the first former Soviet Union country to accede to the WHO Framework Convention on Tobacco Control (FCTG) (2004) with the following adoption of a national tobacco control law to ban smoking in healthcare, education, culture facilities, and public transport. Armenia also banned tobacco advertising on TV and radio (2002) and on billboards (2006), and introduced larger health warnings on cigarette packs. One of the areas where Armenia's progress was less than satisfactory is the FCTC Article 14 on tobacco cessation and treatment. Hence, there is a limited availability of tobacco dependence treatment pharmacotherapy products: only nicotine gum, cytisine and varenicline are registered but are not widely available on the market $^{11}$.

Directly Observed Treatment short-course (DOT) has been the main World Health Organization (WHO) recommendation for treating $\mathrm{TB}$. In Armenia, TB treatment was organized through a healthcare facility-based DOT when the healthcare providers, either a nurse or a physician, supervised the medication administration for at least six months of treatment. The WHO estimated that regular patient-provider contacts and the delivery of smoking cessation interventions to TB patients within a DOT had a potential to reach more than 1 million smokers worldwide and trigger $40 \%$ of TB patients to make a quit attempt ${ }^{12}$. Smoking cessation interventions within regular TB care are feasible, effective, and efficient, and may result in 15-82\% increase in the smoking cessation rate ${ }^{13}$. In addition, through serving as role models and conveying a healthy image, TB healthcare providers might be influential for patients to quit smoking ${ }^{14,15}$. The ABC (Ask, Brief advice and Cessation support) is a simple three-step approach for initiating smoking cessation interventions recommended by the International Union Against Tuberculosis and Lung Disease (known as The Union) for TB patients ${ }^{16}$. The WHO has estimated that up to $67 \%$ of those who receive brief advice, successfully quit at the end of the TB treatment ${ }^{12}$. Provision of brief advice significantly increases the quitting rate $(\mathrm{RR}=1.66)$ compared to no advice provided ${ }^{17}$.

However, in Armenia, healthcare providers do not receive formal training on tobacco dependence treatment during medical education. It is well documented that lack of professional training impacts the provision of tobacco dependence treatment ${ }^{18-20}$. Our study, therefore, aimed to assess TB physicians' knowledge, attitude and practices (KAP), towards smoking cessation within TB care in Armenia to develop targeted interventions and effectively incorporate tobacco dependence treatment within routine $\mathrm{TB}$ services.

\section{METHODS}

This is a qualitative study conducted among TB physicians from Yerevan (the capital city) and two marzes or regions (Syunik and Gegharkunik). We conducted focus group discussions (FGDs) and semi-structured in-depth interviews (IDIs) with physicians from inpatient and outpatient TB clinics who were identified by using a purposive sampling method. Participants were recruited with the help of the National Center of Pulmonology. Semi-structured IDI and FGD guides were developed based on the WHO/The Union 'Monograph on TB and tobacco control' recommendations for joint TB and tobacco control activities 1 and aimed to explore TB physicians' KAP on existing tobacco control activities during both intensive and continuation phases of TB treatment. The guides included domains on knowledge on smoke-free environments, KAP regarding smoking cessation, barriers for provision of smoking counseling and perceived needs for training and future actions. Corresponding questions were developed, agreed upon by the research team and then translated and adapted to the Armenian context.

\section{Data collection and analysis}

The data collection was conducted in summer 2017. The qualitative study followed the research methods of heterogeneity and triangulation, and was terminated when saturation was achieved. The mean duration of the IDIs and FGDs was 32 and 44 minutes, respectively. The sessions were audio-recorded with the permission of all study participants. Each FGD and IDI had a trained moderator and a notetaker. After data collection, the research team transcribed the data and analyzed IDI and FGD transcripts using directed deductive content analysis techniques ${ }^{21}$. TB physicians' practices on the provision of smoking cessation services were analyzed by the ABC smoking 
cessation approach. The study participants were categorized according to the type of clinic (outpatient vs inpatient), the consecutive number of the interview, and location (Yerevan vs marz).

The study was conducted in accordance with the ethical standards of the Institutional Review Board of the American University of Armenia (\#AUA-2017014). Oral informed consent was sought from all the study participants before conducting the interviews. To ensure the confidentiality of the participants and of the collected data, the research team used IDs in all notes and stored data. The data were stored on password-protected computers of the research team allowing access only to its members. Any information provided by the participants that could disclose their identity was excluded from the transcriptions of IDIs and FGDs. Additionally, the study participants were categorized during the data analysis and no personal identifiers were presented next to their statements.

\section{RESULTS}

Overall, 21 physicians participated in five IDIs and four FGDs (two for inpatient services and two for outpatient services). The overwhelming majority of TB physicians were female $(n=19)$ with mean age of 53.43 years (range 27-70 years). Eleven TB physicians worked in inpatient healthcare clinics (Yerevan 1, marzes 10) and 10 worked in outpatient clinics (Yerevan 8, marzes 2). On average, they worked

Table 1. Demographic characteristics of study participants

\begin{tabular}{|c|c|c|}
\hline Characteristics & Categories & $n(\%)$ \\
\hline Age (years), mean (range) & & $53.43(27-70)$ \\
\hline \multirow[t]{2}{*}{ Gender } & Male & $2(9.5)$ \\
\hline & Female & $19(90.5)$ \\
\hline \multirow[t]{3}{*}{ Place of work } & & Inpatient Outpatient \\
\hline & Yerevan & $8(38.1)$ \\
\hline & Marz & $10(47.6)$ \\
\hline \multirow[t]{2}{*}{ Current smoking status } & Smoker & $3(14.3)$ \\
\hline & Non-smoker & 18 (85.7) \\
\hline $\begin{array}{l}\text { Working years as TB } \\
\text { physician, mean (range) }\end{array}$ & & $22.57(3-41)$ \\
\hline \multirow{2}{*}{$\begin{array}{l}\text { Previous participation } \\
\text { in smoking cessation } \\
\text { training }\end{array}$} & Yes & $1(4.8)$ \\
\hline & No & $20(95.2)$ \\
\hline
\end{tabular}

as TB physicians for 22.57 years (range $3-41$ years). Three participants identified themselves as regular smokers. Only one of the TB physicians reported participation in smoking cessation training in the past (Table 1).

\section{Knowledge regarding smoking cessation}

Knowledge regarding the link between $T B$ and tobacco Almost all TB physicians acknowledged that tobacco use was a major problem for their patients and that there was a need for TB patients to quit smoking. A physician from an inpatient TB clinic mentioned:

'... Nowadays the prevalence of chronic obstructive pulmonary disease is growing and one of the causes of the disease is tobacco smoking. Smoking is a big problem for all types of TB, especially, for pulmonary $T B$, because the respiratory tract and the tissues are damaged, and accordingly, the effectiveness of the treatment declines.' (Inpatient TB physician-1, FGD1, marz)

Physicians further identified improved TB treatment effectiveness and outcomes, accelerated recovery, and decreased risk of TB relapse as the most common positive impacts of smoking cessation among pulmonary TB patients. One of the physicians noted:

'Of course smoking status affects the treatment process and outcomes. It [smoking] adversely affects the outcomes making it more difficult to treat patients. Smoking negatively affects TB recurrence, complications, and mortality. I have noticed that.' (Outpatient TB physician, IDI-1, marz)

However, some participants' knowledge of the link between tobacco use and TB treatment outcomes was weak. They were skeptical about the association between smoking and TB and the adverse effects of smoking on TB treatment processes and outcomes. One of the physicians noted:

'I would say that smoking does not have a direct effect on TB. It has an influence on bronchial asthma, cardiovascular disease but not on TB. In general, yes, it somehow influences TB but not directly.' (Outpatient TB physician-3, FGD-3, Yerevan)

According to their perception, smoking did not affect TB treatment, as TB medications were potent enough to treat TB alone:

'I don't think that tobacco use has a significant effect on TB treatment outcomes. Patients receive a huge 
amount of medication that will heal them anyway ...' (Outpatient TB physicians, IDI-2, marz)

Knowledge regarding available smoking cessation methods

Many of the respondents mentioned that they were unaware of specific methods for smoking cessation and available pharmacological therapy. A physician from an inpatient TB clinic mentioned:

'No, we are not [aware of smoking cessation methods] ... I do not know any method.' (Inpatient TB physician-2, FGD-4, marz)

Physicians further elaborated that they do not receive training on tobacco dependence treatment:

'We have never been taught on dependence treatment, we had no such trainings, we do not know the details.' (Outpatient TB physician-2, FGD-3, Yerevan)

The majority of physicians reported that in their practice, they applied only 'psychological' methods (referring to non-formal behavioral counselling) to convince their patients to quit.

'Only psychological [behavioral counseling. methods]. The patients themselves should be prepared to either decrease smoking or quit abruptly.' (Outpatient TB physician-2, FGD-3, Yerevan)

Another physician said:

'I have heard that there were some gums but I do not know about their effectiveness. There was also cytisine in the form of tablets. The only method I know is psychological [non-formal behavioral counselling methods].' (Outpatient TB physician-1, FGD-2, Yerevan)

Some respondents listed smoking cessation methods including nicotine replacement therapy (NRT) (gum and patches), electronic cigarettes, and tablets; however, they had doubts about the effectiveness of these methods. Many physicians emphasized the overarching role of willpower of a patient in quitting smoking and said:

'If the patients are not willing to quit, no medications will help.' (Outpatient TB physician-2, FGD-3, Yerevan)

\section{Attitude regarding smoking cessation}

Non-appropriateness of smoking cessation interventions While discussing the possibility of implementing smoking cessation interventions within the standard TB treatment regimen, physicians were concerned about the appropriateness of the pharmacological interventions for smoking cessation specifically for TB patients. Drug interactions between smoking cessation and TB medications, the possible side effects of smoking cessation pharmacotherapy and patients' resistance to taking smoking cessation pharmacotherapy in addition to TB medications were mentioned as major challenges for providing tobacco dependence treatment. Physicians noted:

'It depends on toxicity of those [smoking cessation products] medications. For example, our [TB treatment medications] medications have many side effects on the liver ... on all other organ systems ... So, we should know that [drug interactions], in order not to worsen the condition.' (Outpatient TB physician-1, FGD-2, Yerevan)

'If the [tobacco dependence] treatment is pharmacological; we would not offer it to our patients. Our patients receive many medications. Even if they have pain, we cannot prescribe painkiller or vitamin, they do not want to take it, because they take a large number of medications.' (Outpatient TB physician-3, FGD-3, Yerevan)

Perceived challenges of smoking cessation interventions for TB patients

TB physicians revealed various challenges that impacted their determination to implement smoking cessation interventions with their patients. The majority of physicians believed that the provision of smoking cessation interventions to TB patients will pose an additional burden to them and they did not consider such interventions as their immediate duty. Additionally, physicians named other professionals who should be in-charge for provision of smoking cessation interventions (e.g. psychologists, epidemiologists, and social workers). Physicians from an inpatient TB clinic mentioned:

'This is not our job. Of course, this is more educational intervention [not medical treatment] which should be carried out by epidemiologists, psychologists, social workers ... but not by us definitely.' (Inpatient TB physician-3, FGD-1, marz)

'It is not my business, considering that I cannot address this issue as a specialist. Shoemaker should sew shoe.' (Inpatient TB physician-2, FGD-1, marz)

Furthermore, physicians believed that throughout the treatment their patients faced an abundance of 
restrictions, and forcing patients to quit smoking would add extra stress on them. This was reflected in the physician's statement:

'Do not get into contact with people, do not use alcohol, do not smoke ... We have so many "Do not's" that we already do not know which one to emphasize. They have so many restrictions that they go into psychological shock. If we emphasize quitting smoking so much, the patient will be in very poor condition.' (Outpatient TB physician-3, FGD-3, Yerevan)

Some physicians listed psychological distress (which patients experience upon discovering their diagnosis), the anxiety because of the nature of TB treatment (long duration, fear of infecting others, and isolation), withdrawal symptoms, and reluctance to accept pharmacological interventions within the scope of TB treatment as threats to effective smoking cessation interventions. One of the physicians from Yerevan noted:

'TB patients are already stressed and depressed, they are scared of infecting their family members, they visit their relatives less frequently, and the relatives themselves visit them less frequently. These all have an influence on them and those stressful conditions make them smoke.' (Outpatient TB physician-1, FGD-2, Yerevan)

Another inpatient TB physician mentioned:

'First, they have TB and they are highly stressed about that. This is a big problem. Second, they are isolated from their social environment. These factors lead patients to continue smoking or even to smoke more.'(Inpatient TB physician-1, FGD-1, marz)

Perceived opportunities for smoking cessation interventions for TB patients

Despite the challenges, physicians also identified some opportunities that the diagnosis of TB could bear in terms of quitting smoking. TB physicians mentioned that after being diagnosed with TB and admitted to an inpatient TB clinic, some patients wish to quit smoking.

'I had cases when patients came and said that they had quit smoking right after starting the TB treatment ... Although they smoked for many years, they quit smoking when they started to take TB medications.' (Inpatient TB physician-1, FGD-4, marz)

Furthermore, TB physicians noted that during the continuous patient-provider encounters, patients demonstrated strong trust in TB physicians, which might be used as an opportunity to engage the patients in smoking cessation counselling. This idea was supported by many physicians:

"When patients come to us, they expect us to authoritatively dictate how they should "breathe". Our role is huge, and we need to tell them what will happen, if they continue to smoke or quit smoking. Our role is to present the complications adequately.' (Outpatient TB physician-2, FGD-2, Yerevan)

'I have noticed that my patients trust me a lot. This is very important. Patients come and tell me: "Doctor, I will do whatever you tell me". It is a huge thing.' (Outpatient TB physician, IDI-2, marz)

According to physicians, smoking female TB patients were less receptive to smoking cessation interventions compared to male smokers. One physician from Yerevan said:

'I noticed that smoker women are more depressed. For example, I had a patient who said that she will die if not smoking. Maybe they reduce smoking a little from 10 cigarettes to 7 or 8 and that is all.' (Outpatient TB physician-3, FGD-2, Yerevan)

\section{Practice regarding smoking cessation}

Physicians described some basic actions they routinely implement concerning smoking cessation. During their routine practice, TB physicians asked about their TB patients' smoking status and recorded the responses in the medical records and the electronic database. Some physicians also asked about the duration and number of cigarettes smoked per day. One of the outpatient clinic physicians said:

'Of course, we ask. We ask about the duration of smoking and the daily number of smoked cigarettes.' (Outpatient TB physician-1, FGD-2, Yerevan)

Asking the smoking status was often followed by provision of brief advice on quitting smoking through emphasizing the harmful effects of smoking on the TB treatment process and the potential complications of treatment in case of not quitting smoking. According to the physicians:

'They [TB patients] may say that it is difficult to quit and I explain to all of them that smoking is very harmful and is a risk factor for this disease [TB]. We also explain that the treatment process will be difficult and there might be problems in the future.' (Outpatient TB physician-4, FGD-3, Yerevan) 
'Our patients are different from each other and that is why everything is very individual. However, we talk to every patient about the harms of smoking, except those who have already quit.' (Inpatient TB physician-1, FGD-1, marz)

The overwhelming majority of TB physicians reported feeling confident in advising patients to quit and further added that for TB patients the smoking cessation advice should be provided in a soft and non-judgmental manner to prevent patients from becoming even more depressed. Physicians listed several behavioral and psychological techniques they offered to smoking TB patients as cessation support. TB physicians commonly used 'scaring' strategies to motivate patients to make a quit attempt. According to physicians:

'Sometimes we show their $x$-rays and the patients see those "holes", as they used to say, and it is very impressive.' (Inpatient TB physician-2, FGD-1, marz)

'We can just scare them, some of them will be scared and will quit, and some will not ...' (Outpatient TB physician-1, FGD-2, Yerevan)

For those patients, who were willing to quit they were usually offered behavioral tips (sunflower seeds, chewing gum, or candies) to help them to cope with the withdrawal symptoms. Some physicians mentioned advising their patients to reduce the number of smoked cigarettes, especially for those who failed to quit. One physician stated:

'There are patients who ask to provide some smoking. cessation medications to overcome dependence faster. I suggest using sweets, gums [not nicotine], or something else. At that moment they can chew those gums for 5 to 10 minutes, overcome that situation and forget about smoking or skip the next several cigarettes. That is also an option.' (Outpatient TB physician-3, FGD-2 Yerevan)

The vast majority of $\mathrm{TB}$ physicians reported not prescribing smoking cessation medication to their patients because of lack of knowledge and experience on tobacco dependence treatment, distrust towards the effectiveness of pharmacotherapy, and possible interactions with TB medication.

'I don't know how those medications will interact with TB treatment. These medications for tobacco dependence treatment may harm patients even more. This is why patients should continue smoking rather than quitting.' (Inpatient TB physician, IDI-3, marz)

Most physicians reported varying practices for smoking cessation and attributed it to the absence of a universal algorithm and formal tobacco dependence treatment protocols for TB patients. According to them, that impedes the provision of smoking cessation assistance to TB patients. One physician mentioned:

'There is no special algorithm to talk to the patient. I have my approach, the other physician has hers/his, and so on. Our patients are different from each other and that is why everything is very individual. However, we talk to every patient about the harms of smoking, except those who have already quit.' (Inpatient TB physician-1, FGD-1, marz)

According to another physician:

'It should be included in the [TB treatment] scheme [approved by the MOH]. For each patient, we have separate schemes. We need to include smoking cessation medications there for those smokers, who would not be able to quit smoking by psychological interventions or on his/her own. Otherwise, we cannot prescribe medications.' (Outpatient TB physician-4, FGD-2, Yerevan)

\section{DISCUSSION}

The study explored TB physicians' knowledge, attitudes and practices with regard to provision of smoking cessation assistance to their patients within DOT. None of the participants, except one, had formal training on smoking cessation since it is not included in the curriculum of the formal medical education in Armenia. Some of the medical professionals learn about health hazards of smoking and smoking cessation counseling during local and international postgraduate training programs. Not all of the physicians were aware of the link between smoking and TB and the available smoking cessation methods, which resulted in a negligent attitude towards the importance of implementing smoking cessation interventions and inappropriate practices. TB physicians usually asked about smoking status and provided brief advice to their patients. Despite the fact that a few physicians were able to list some of the existing pharmacotherapy products and behavioral tips for smoking cessation, most of the indicated strategies were beyond the evidence-based tobacco dependence treatment recommendations. 
Various studies identified that inadequate knowledge and skills were provider-level barriers to practicing smoking cessation interventions ${ }^{22-25}$. In contrast, the presence of previous training on smoking cessation could significantly improve the healthcare providers' practice scores $^{15,26}$.

Additional to the lack of knowledge and skills, the interviewed TB physicians did not prioritize smoking cessation during their routine activities, as the TB treatment was mentioned as their primary responsibility. A study conducted by Vogt et al. ${ }^{27}$ also reported the physicians' perception that the provision of smoking cessation assistance was outside the scope of their professional responsibilities was a factor that could impede discussing smoking cessation with patients.

Though the majority of study participants emphasized possible drug interactions between smoking cessation pharmacotherapy and TB medications as a barrier to prescribing medicinal treatment for tobacco dependence, a clinical trial by Sharma et al..$^{28}$ that explored the effectiveness of pharmacotherapy versus behavioral counseling in addition to DOT did not report any drug interactions. The study further revealed that biochemical smoking quit rates and self-reported quit rates were significantly higher in the arm that received NRT.

Some physicians acknowledged that the diagnosis of TB was a key teachable moment, as patients diagnosed with lung disease were more receptive to physicians' advice to quit. This finding was also consistent with the literature suggesting that TB patients might be highly motivated to quit smoking ${ }^{29}$. A study conducted by Aryanpur et al. ${ }^{29}$ reported that at the time of TB diagnosis, $23.8 \%$ of newly diagnosed smoking TB patients quit immediately and $52.4 \%$ of patients who continued to smoke had intention to quit during the following month. Other studies also confirmed that the diagnosis of TB was an opportunity for initiating smoking cessation interventions, suggesting that smoking cessation should be adopted into TB care to ensure a successful integration of TB and tobacco control activities ${ }^{30-31}$. The finding indicating that women are less receptive to smoking cessation compared to men was consistent with the literature ${ }^{32-35}$; however, in our study this phenomenon was discussed as an opportunity for initiating smoking cessation interventions specifically among Armenian TB patients who are, in fact, predominantly men ${ }^{10}$.

\section{Strengths and limitations}

The qualitative assessment of TB physicians' KAP allowed us to deeply explore their knowledge gaps, perceptions and practices with regard to provision of smoking cessation interventions. We targeted both inpatient and outpatient clinic TB physicians to learn their experiences in implementing smoking cessation interventions with patients receiving intensive and continuation phases of TB treatment. The study findings may have low generalizability to all TB healthcare providers as only physicians' perspectives have been explored. Studies also exploring TB nurses' and TB patients' perspectives could offer a wider understanding of how to incorporate smoking cessation interventions within TB care in Armenia.

\section{CONCLUSIONS}

Our findings suggest that TB physicians had insufficient knowledge and skills on smoking cessation and inconsistent attitudes towards the importance of implementing smoking cessation interventions among TB patients. Tobacco dependence treatment should be formally incorporated into the national TB treatment strategy to support and enforce the provision of tobacco dependence treatment to all TB patients in Armenia. In addition, the study findings might be relevant to other countries with similar healthcare contexts as well as TB and tobacco profiles. Therefore, the findings on TB physicians' knowledge, attitude and practice might have practical implications for designing targeted interventions, both locally and globally, for advancement of joint TB and tobacco control efforts in accordance with international recommendations.

\section{REFERENCES}

1. World Health Organization. The Union monograph on TB and tobacco control: joining efforts to control. Geneva, Switzerland: World Health Organization; 2007. https://apps.who.int/iris/bitstream/handle/10665/43812/ 9789241596220_eng.pdf?sequence $=1$ \&isAllowed $=y$. Accessed November 1, 2020.

2. Slama K, Chiang CY, Enarson DA, et al. Tobacco and tuberculosis: A qualitative systematic review and metaanalysis. Int J Tuberc Lung Dis. 2007;11(10):1049-1061. PMID:17945060. 
3. Lin HH, Ezzati M, Chang HY, Murray M. Association between tobacco smoking and active tuberculosis in Taiwan: Prospective cohort study. Am J Respir Crit Care Med. 2009;180(5):475-480. doi:10.1164/rccm.200904-05490C

4. Bates MN, Khalakdina A, Pai M, Chang L, Lessa F, Smith KR. Risk of tuberculosis from exposure to tobacco smoke: a systematic review and meta-analysis. Arch Intern Med. 2007;167(4):335-342. doi:10.1001/archinte.167.4.335

5. Jeyashree K, Kathirvel S, Hd S, Kaur H, Goel S. Smoking cessation interventions for pulmonary tuberculosis treatment outcomes (Review). Cochrane Database Syst Rev. 2016;(1):CD011125. doi:10.1002/14651858.CD011125.pub2

6. Harutyunyan A, Abrahamyan A, Hayrumyan V, Grigoryan Z, Truzyan N, Petrosyan V. Knowledge, Attitude and Practice towards Tobacco Control Activities within Tuberculosis Services in Armenia: A Qualitative Study. Yerevan, Armenia: Turpanjian School of Public Health, American University of Armenia; 2018. https://chsr.aua. am/files/2018/07/Knowledge-Attitude-and-Practicetowards-Tobacco-Control-Activities-within-TuberculosisServices-in-Armenia.pdf. Accessed December 13, 2020.

7. Drope J, Schluger N, Cahn Z, et al. Comorbidities. The Tobacco Atlas. Atlanta, GA: American Cancer Society and Vital Strategies; 2018. https://tobaccoatlas.org/topic/ comorbidities/. Accessed November 17, 2020

8. World Health Organization. Use of high burden country lists for TB by WHO in the post-2015 era. Geneva, Switzerland: World Health Organization; 2015. https:// www.who.int/tb/publications/global_report/high_tb_ burdencountrylists2016-2020.pdf. Accessed November 1, 2020.

9. World Health Organization. Global Tuberculosis Report 2019. Geneva, Switzerland: World Health Organization; 2019. https://www.who.int/publications/i/item/globaltuberculosis-report-2019. Accessed November 1, 2020.

10. Khachadourian V, Truzyan N, Harutyunyan A, et al. People-centred care versus clinic-based DOT for continuation phase TB treatment in Armenia: A cluster randomized trial. BMC Pulm Med. 2020;20(1):1-10. doi:10.1186/s12890-020-1141-y

11. Harutyunyan A. Availability, Affordability, and Prices of Smoking Cessation Products in 9 Countries: Preliminary Findings. Tob Prev Cessation. 2016;2(April Supplement):21. doi:10.18332/tpc/62413

12. World Health Organisation. Toolkit for delivering the 5A's and 5R's brief tobacco interventions to TB patients in primary care. Geneva, Switzerland: World Health Organization; 2014. https://apps.who.int/iris/ bitstream/handle/10665/112836/9789241506946_eng. pdf?sequence=1. Accessed November 1, 2020.

13. Whitehouse E, Lai J, Golub JE, Farley JE. A systematic review of the effectiveness of smoking cessation interventions among patients with tuberculosis. Public Health Action. 2018;I(2):37-49. doi:10.5588/pha.18.0006 14. World Health Organisation. The role of health professionals in tobacco control. Geneva, Switzerland: World Health Organization; 2005. https://www.who. int/tobacco/resources/publications/wntd/2005/ bookletfinal_20april.pdf. Accessed November 1, 2020.

15. Gichuki JW, Opiyo R, Mugyenyi P, Namusisi K. Healthcare providers' level of involvement in provision of smoking cessation interventions in public health facilities in Kenya. J Public Health Africa. 2016;6(2):6267. doi:10.4081/jphia.2015.523

16. Bissel K, Fraser T, Chiang CY, Enarson DA. Smoking cessation and smokefree environments for tuberculosis patients. Paris, France: International Union Against Tuberculosis and Lung Disease; 2010. https://theunion. org/sites/default/files/2020-08/pub_smokingcessation_ eng.pdf. Accessed November 1, 2020.

17. Stead LF, Buitrago D, Preciado N, Sanchez G, HartmannBoyce J, Lancaster T. Physician advice for smoking cessation. Cochrane Database Syst Rev. 2013(5):CD000165. doi:10.1002/14651858.CD000165.pub4

18. Abdullah SM, Husten CG. Promotion of smoking cessation in developing countries: A framework for urgent public health interventions. Thorax. 2004;59(7):623-630. doi:10.1136/thx.2003.018820

19. Jradi H. Awareness, Practices, and Barriers regarding Smoking Cessation Treatment among Physicians in Saudi Arabia. J Addict Dis. 2017;36(1):53-59. doi:10.1080/10550887.2015.1116355

20. Harutyunyan A, Abrahamyan A, Hayrumyan V. Perceived barriers of tobacco dependence treatment: a mixedmethods study among primary healthcare physicians in Armenia. Prim Health Care Res Dev. 2019;20:e17. doi:10.1017/S1463423618000828

21. Hsieh H, Shannon SE. Three approaches to qualitative content analysis. Qual Health Res. 2005;15(9):12771288. doi:10.1177/1049732305276687

22. Panaitescu C, Moffat MA, Williams S, et al. Barriers to the provision of smoking cessation assistance: A qualitative study among Romanian family physicians. NPJ Prim Care Respir Med. 2014;24(May):14022. doi:10.1038/npjpcrm.2014.22

23. Meijer E, Van Der Kleij RMJJ, Chavannes NH. Facilitating smoking cessation in patients who smoke: A large-scale cross-sectional comparison of fourteen groups of healthcare providers. BMC Health Serv Res. 2019;19(1):1-16. doi:10.1186/s12913-019-4527-x

24. Desalu OO, Adekoya AO, Elegbede AO, Dosunmu A, Kolawole TF, Nwogu KC. Knowledge of and practices related to smoking cessation among physicians in Nigeria. J Bras Pneumol. 2009;35(12):1198-1203. doi:10.1590/S1806-37132009001200006

25. Yan J, Xiao S, Ouyang D, Jiang D, He C, Yi S. Smoking behavior, knowledge, attitudes and practice among health care providers in Changsha city, China. Nicotine Tob Res. 2008;10(4):737-744. doi:10.1080/14622200801901930

26. Cornuz J, Humair JP, Seematter L, et al. Efficacy of 
resident training in smoking cessation: A randomized, controlled trial of a program based on application of behavioral theory and practice with standardized patients. Ann Intern Med. 2002;136(6):429-437. doi:10.7326/0003-4819-136-6-200203190-00006

27. Vogt F, Hall S, Marteau TM. General practitioners' and family physicians' negative beliefs and attitudes towards discussing smoking cessation with patients: A systematic review. Addiction. 2005;100(10):1423-1431. doi:10.1111/j.1360-0443.2005.01221.x

28. Sharma SK, Mohan A, Singh AD, et al. Impact of nicotine replacement therapy as an adjunct to anti-tuberculosis treatment and behaviour change counselling in newly diagnosed pulmonary tuberculosis patients: An openlabel, randomised controlled trial. Sci Rep. 2018;8(1):19. doi:10.1038/s41598-018-26990-5

29. Aryanpur M, Masjedi MR, Mortaz E, et al. Intention to quit smoking and associated factors in smokers newly diagnosed with pulmonary tuberculosis. Tanaffos. 2016;15(1):17-24. PMID:27403174.

30. Shin SS, Xiao D, Cao M, et al. Patient and doctor perspectives on incorporating smoking cessation into tuberculosis care in Beijing, China. Int J Tuberc Lung Dis. 2012;16(1):126-131. doi:10.5588/ijtld.11.0044

31. Zvolska K, Pankova A, Nohavova I, et al. A narrative review of facilitators and barriers to smoking cessation and tobacco-dependence treatment in patients with tuberculosis in low- and middle-income countries. Tob Induc Dis. 2020;18(August). doi:10.18332/tid/125195

32. Rahmanian SD, Diaz PT, Wewers ME. Tobacco use and cessation among women: Research and treatmentrelated issues. J Women's Heal. 2011;20(3):349-357. doi:10.1089/jwh.2010.2173

33. Allen AM, Oncken C, Hatsukami D. Women and Smoking: The Effect of Gender on the Epidemiology, Health Effects, and Cessation of Smoking. Curr Addict Reports. 2014;1(1):53-60. doi:10.1007/s40429-013-0003-6

34. Chaney SE, Sheriff SW, Merritt L. Gender differences in smoking behavior and cessation. Clin Nurs Stud. 2015;3(3):17-22. doi:10.5430/cns.v3n3p17

35. Karadoğan D, Önal Ö, Kanbay Y. How does reimbursement status affect smoking cessation interventions? A real-life experience from the Eastern Black Sea region of Turkey. Tob Induc Dis. 2019;17(January). doi:10.18332/tid/100412
CONFLICTS OF INTEREST

The authors have completed and submitted the ICMJE Form for Disclosure of Potential Conflicts of Interest and none was reported.

\section{FUNDING}

This work was supported by a grant from Global Bridges Healthcare Alliance for Tobacco Dependence Treatment, hosted by Mayo Clinic and Pfizer Independent Grants for Learning and Change.

PROVENANCE AND PEER REVIEW

Not commissioned; externally peer reviewed. 